EPJ Web of Conferences 87,03004 (2015)

DOI: $10.1051 /$ epjconf/ 20158703004

(C) Owned by the authors, published by EDP Sciences, 2015

\title{
Computational studies on ECE spectrum for ITER, in the presence of a small fraction of non-thermals and radial resolution evolution for oblique view
}

\author{
P.V. Subhash ${ }^{1, a}$, Yashika Ghai ${ }^{2}$, Hitesh K. Pandya ${ }^{1}$, Amit K. Singh ${ }^{1}$, A. M. Begam ${ }^{3}$, and P. Vasu ${ }^{1}$ \\ 1 ITER-India, Institute for Plasma Research, Gandhinagar, India \\ ${ }^{2}$ GNDU, Amritsar, Punjab, India \\ ${ }^{3}$ Kanchi Mamunivar Center for Post Graduate Studies, Poducherry, India
}

\begin{abstract}
In tokamaks, the temperature measurement using different techniques like Electron Cyclotron Emission (ECE), Thomson scattering etc. shows differences because of various phenomena. The physical reasons for this are not entirely understood. Thus to have comprehensive understanding of these difference, the contribution from each phenomenon needs to be individually understood. The phenomenon affecting radial temperature profile measurement includes harmonics overlap, relativistic down shifting, presence of non-thermals etc. For ITER like plasma, radial temperature profiles can be obtained from the first harmonics ordinary $(\mathrm{O})$ mode or second harmonic extra-ordinary $(\mathrm{X})$ mode of ECE spectrum. It is possible that, higher harmonics produced from the non-thermals can be relativistically downshifted to second harmonics and results a deviation in the measured temperature profile. We performed a parametric study on the e ffect of non-thermal electrons on measured ECE temperature for ITER scenario-2. All the numerical calculations reported in this paper are performed using NOTEC computer code which is capable of handling non-thermal populations. After proper validation of numerical methods using normal electron population (without non-thermals) a parametric study with non-thermals is performed. In the parametric study radial locations of non-thermals, energy of non-thermals and fraction of non-thermals are considered. This study is initially performed for normal view and later extended in to oblique views. The range of deviation of temperature over the examined parametric regime as well as the possible physical reasons will be presented. The effect of parallel component of non-thermal energy is also examined. Finally results of one set of study for oblique view (where the detector is not exactly normal to the magnetic field) with non-thermal electrons are also presented. In ITER apart from an Electron Cyclotron Emission (ECE) detector placed normal to magnetic field an oblique view detector is planned to grab information about non-thermal electrons. Usefulness of such an additional detector for a better radial resolution is examined. The differences in the ECE spectrum from a tokamak plasma between a direct LOS (normal to toroidal magnetic field) and a slightly oblique LOS have been modelled. A typical ITER tokamak scenario has been chosen in this study. The intensities of radiation, as observable from the low-field side, covering the first harmonic O-mode spectral frequencies $105-230 \mathrm{GHz}$ have been compared. The physical reasons for the code-predicted results, regarding the differences between the direct and oblique spectra, are elucidated. Finally, signatures of the presence of non-thermals from a comparison of normal view and oblique view are also examined.
\end{abstract}

\section{Introduction}

Electron Cyclotron Emission (ECE) measurement is considered as one of the reliable techniques for radial electron temperature $\left(T_{e}\right)$ measurement in tokamaks $[1,2]$. A comprehensive review of physical and technical aspects are available from reference [1]. The frequency of emission of ECE along the radial location can be calculated by using the local toroidal magnetic field strength in the corresponding locations. By measuring the ECE intensity for many relevant frequencies radial temperature profile can be obtained. The procedure of obtaining $T_{e}$ through ECE measurement is well established in literature (See ref $[1,2]$ and references therein). For ITER the radial temperature information can be inferred from first harmonics ordinary modes (O-modes) or second harmonics extra ordinary modes (X-modes) [2, 3]. But for high temperature tokamaks like ITER the capabilities of this method is limited by various effects like harmonic overlap, polarization scrambling, due to the deviation of electron populations from Maxwellian etc [4].

Many studies are reported on difference between measured temperature using ECE and other techniques like Thomson Scattering(TS). G. Taylor reported a difference of 10-20\% between ECE and TS when heated with NBI at $10 \mathrm{KeV}$ electron temperature [5]. JET with NBI heating alone or with combination of ICRH found a discrepancy about $30 \%$ at $12 \mathrm{KeV}$ [6]. Recent experiments on JET to understand the discrepancy on heating method finds that some methods like ICRF do not show any discrepancy [7]. Another experiments at DIII-D and C-MOD

\footnotetext{
ae-mail: subhashpv@iter-india.org
} 
with electron temperature up to $8 \mathrm{KeV}$ found out no obvious difference [8]. Hence it can be concluded that the difference in temperature measurement between ECE and other methods strongly depend up on heating methods as well as electron temperature. This is not well understood. ITER is expected to reach a temperature up to $25 \mathrm{KeV}$ with strong electron-ion coupling. It is important to understand the 'deviation' in the ECE measurement individually for direct electron heating (such as ECCD and ECRH) and ion heating (such as ICRH and NBI). Departure of the electron distribution from Maxwellian is considered as one possible cause of this deviation. The electron populations can be deviated from Maxwellian because of the presence of super thermal electrons.

\section{Radial resolution evaluation}

\subsection{Normal view}

The calculations reported in this article are performed using a computer code NOTEC (NOn Thermal ECE). NOTEC code calculates measured ECE spectra using antenna pattern, reflection, refraction and non-thermal populations [9, 10].A burning H-mode ITER scenario-2 [11, 12] has been taken for these calculations. The major and minor radius of the ITER machine is $6.2 \mathrm{~m}$ and $2 \mathrm{~m}$ respectively. The detector is assumed to be at radius $\mathrm{R}=8.2$ $\mathrm{m}, \mathrm{Z}=0.76 \mathrm{~m}$ and $\phi=0$.

For ITER, in principle the radial temperature can be deduced from first harmonics ordinary mode ( $\mathrm{O}$ mode) and second harmonics extra ordinary mode (X-mode). Such

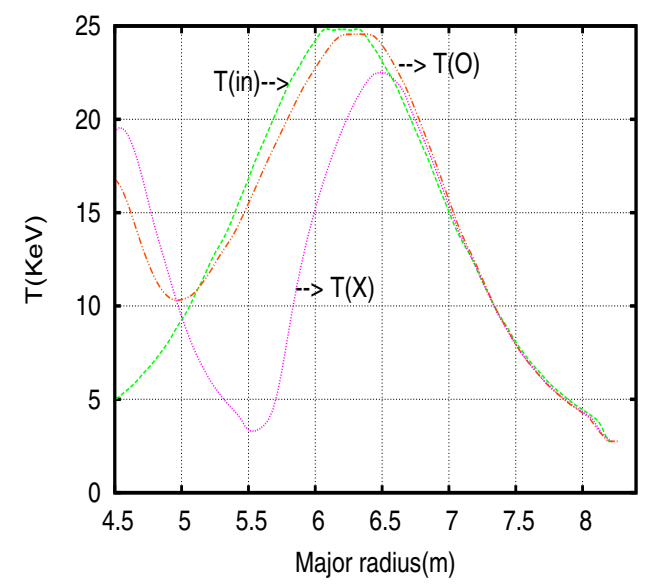

Figure 1. The input(T(in)) and calculated (with O-mode $(\mathrm{T}(\mathrm{O}))$ and $\mathrm{X}$-mode $(\mathrm{T}(\mathrm{X})))$ temperature profiles.

kind of temperature profiles are calculated using NOTEC computer code. For the purpose of all the calculations reported in this article the ITER parameters and profiles are adapted from Ref [13]. The input and calculated temperature profile from first harmonics $\mathrm{O}$-mode and second harmonics X-mode is shown in figure 1.

The temperature calculated in the present study is in good agreement with previous studies reported in [4, 14].
From figure 1 it is clear that O-mode yields more reliable temperature profile can be obtained. Hence most of the calculations reported in this article is limited to O-mode unless otherwise specified.

\subsection{Oblique view}

In similar way, calculations are repeated for three cases of oblique view detector. Here the detector is assumed to be placed in an angle with respect to the normal to the magnetic field in the toroidal plane. The three cases simulated here are for the angles $5^{\circ}, 10^{\circ}$ and $20^{\circ}$.The obtained frequency spectrum for all three cases along with the spectrum for normal view case is shown in figure 2. From the figure 2 it is clear that frequency spectrum for all the oblique cases are shifted from normal view spectrum. Further the amount of the shift is different for different viewing angles. The frequency shift is due to Doppler shift, which modify the resonance condition into:

$$
\omega=n \frac{e B}{m_{e}\left(\gamma-N_{\|} u_{\|}\right)}
$$

Where $N_{\|}$is the parallel component of refractive index and $u_{\|} \equiv P / m_{0} c$ is parallel component of normalized electron momentum.

The usefulness of equation 1 is limited as $N_{\|}$is functions of local plasma parameters. But the NOTEC code is capable to provide the location of emission for any frequency. This has been used for obtaining one to one correspondence between location of emission and frequency. The corresponding plot for the location of emission against frequency is shown in figure 3 . Figure 3 is used to obtain the radial temperature profile for all the cases. In an experimental situation also one need to adopt some methods to counter the shift in the oblique spectrum to obtain radial temperature profile. This is can be achieved either by using numerical calculations or by locating the phenomena (for example fluctuations) from the normal view and extract the properties of phenomena from both type of views. The second methodology is relevant only for understanding fluctuations or instabilities.

Following important points can be noted from figure 2 and figure 3 . For the case of oblique view same frequency is emitted from a different radial location when comparing with normal view. Any change in the angle of view, results different location of emission for the same frequency. The extend of radial emission for a particular frequency is determined by the local properties of plasma through emission and absorption coefficients. Further, the amount (intensity) of the signal received by the detector will be determined by the properties of the plasma along the 'path of the ray' between the location of emission and detector position. Hence the absorption or amplification of signal through fundamental or higher harmonics along the ray path for normal and oblique view will be different. Hence it can be expected that in principle, the radial resolution for oblique view can be different from normal view. This point is further studied in the next section. 


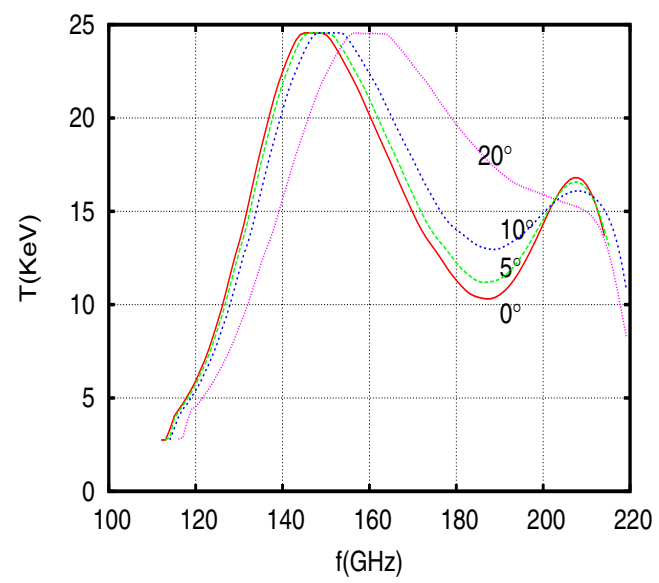

Figure 2. Obtained frequency spectrum for frequencies between $112 \mathrm{GHz}$ to $220 \mathrm{GHz}$ for various viewing angle.

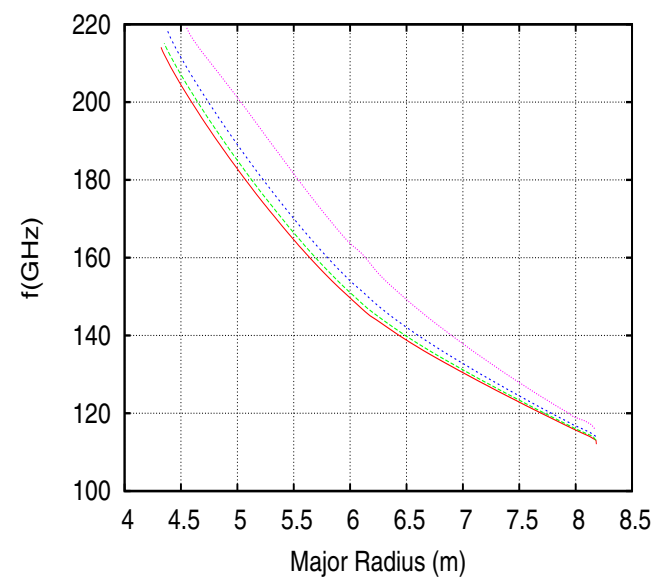

Figure 3. The one to one correspondence between location of emission and frequency for various viewing angle. The curves from bottom to top are for $0^{\circ}, 5^{\circ}, 10^{\circ}$ and $20^{\circ}$ respectively.

\section{Dependence of radial resolution and localization of fluctuations on viewing angle of ECE detector}

\subsection{Radial resolution}

The radial resolution of ECE emission for a particular frequency for the purpose of the calculations reported here is defined as the radial thickness of emission between $5 \%$ to $95 \%$ of the total intensity. In order to demonstrate this point we have shown an example of calculating width of emission $(\delta r)$ for a frequency $\mathrm{f}=142 \mathrm{GHz}$ for the case of normal view in figure 4 . From the figure 4 it is clear that the emission for this particular frequency is centered around a radial location of 6.3 and extent of emission (between $5 \%$ to $95 \%$ ) is between $6.245 \mathrm{~m}$ and 6.360 . Hence the radial resolution of emission $(\delta r)$ for $142 \mathrm{GHz}$ is calculated as $11.5 \mathrm{~cm}$. This process is repeated for all the frequencies ranging from $122 \mathrm{GHz}$ to $220 \mathrm{GHz}$ for normal

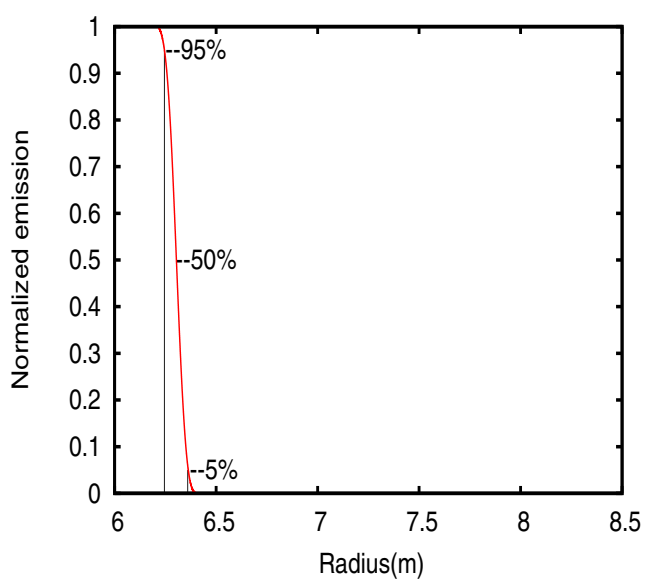

Figure 4. Normalized emission as a function of radius for $\mathrm{f}=142 \mathrm{GHz}$.

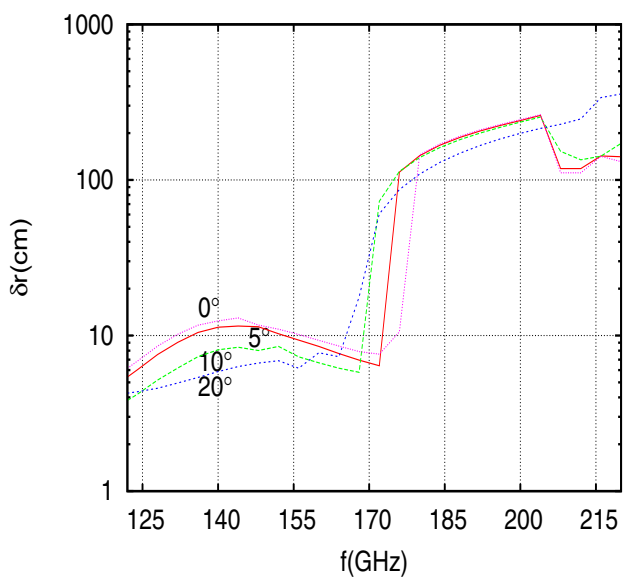

Figure 5. Radial resolution for frequencies from $112 \mathrm{GHz}$ to 220 $\mathrm{GHz}$ for various viewing angle.

view and three cases of oblique view $\left(5^{\circ}, 10^{\circ}\right.$ and 20 $\left.{ }^{\circ}\right)$.The selected frequency range almost cover the entire first harmonic O-mode. The radial resolution for all the frequencies in the first harmonics are shown in figure 5. The range of selected frequencies almost cover the entire tokamak in terms of location of emission. Following interesting points can be obtained from the figure 5 after dividing the frequencies in to four region. The frequencies between $122 \mathrm{GHz}$ and $170 \mathrm{GHz}$ are coming from relatively narrow region (of few centimeters) for all the four cases. For the case of normal view the radial resolution is ranging from $5.5 \mathrm{~cm}$ to $13 \mathrm{~cm}$. The maximum is found to be around $144 \mathrm{GHz}$. While for the $20^{\circ}$ case the radial resolution $\delta r$ is ranging from $4.4 \mathrm{~cm}$ to $7.7 \mathrm{~cm}$. The other two cases are fitted in between these two views. So for this region the oblique view case clearly shows a better radial resolution. For example, for the $20^{\circ}$ case $\delta r$ is less than half of the normal case around $140 \mathrm{GHz}$. It should also be noted that the radial resolution improve with an increase in viewing angle through this region. This region is im- 
portant as it cover the entire inboard side and center of the plasma.

\section{Effect of a small amount of non-thermal populations on T measurement for ITER}

\subsection{Parametric regime}

In the parametric study reported here, the base profiles are seeded with super thermal electrons.

In order to include super thermals in the numerical scheme, we need to know location of the super thermals, energy of the super thermals and fraction of the super thermals relative to the background electron density. Ideally the above parameters can be obtained by solving FokkerPlank equation for the corresponding heating or current drive processes, which in itself is a separate problem and is beyond the scope of this work. Nevertheless, we can understand effect of super thermals on measured spectrum in a parametric study over a parametric regime of relevance. Such a study also provide insights of various physical aspects of the problem within the frame work of the scanned parametric space. The present work attempt this method.

Three parameters considered in this study are location of the super thermals, fraction of the super thermals and perpendicular component of the energy of the super thermals.

We performed the calculations for three radial locations which correspond to safety factor $\mathrm{q}=1, \mathrm{q}=1.5$ and $q=2$. These locations are selected because the proposed ECCD to stabilize various instabilities will be used at these locations. The super thermals are assumed to be distributed in a drifted Maxwellian form [9] between the flux tubes bounded by $3 \%$ around various 'q' values and is further limited to a poloidal extend of 0.14 radian in the line of sight for the normal view. The fraction of super thermals are varied from $0.05 \%$ to $0.5 \%$. As we see later in this article any amount of super thermal electrons lower than 0.05 has no effect on the temperature measurement. The upper limit of the of super thermals are set to $0.5 \%$ because logically we are not expecting more than this value. Even in JET the maximum possible fraction is about 0.12 $\%$ (see reference [15]). So at present we do not expect super thermal electrons more than $0.5 \%$ in ITER. The energy range of super thermals are initially bracketed in a range between $100 \mathrm{KeV}$ and $250 \mathrm{KeV}$.

\subsection{Results of parametric study}

In this section following results are included. Initially, results of the parametric study is presented. This is followed by results for oblique view, where detector is placed at an angle with the magnetic field.

The effect of super thermals are analyzed in following way. Initial calculations are performed with-out super thermal to obtain the frequency spectrum. This is then followed by calculating spectrum with the inclusion of super thermals for the entire range of parametric regime. The obtained spectrum without super thermals are then subtracted from the spectrum with super thermals to obtain the deviation as the function of frequencies and radial locations. We call the difference of intensities with and without super thermals as 'deviation' throughout rest of this article. The emission for the both first and second harmonics for ITER is relatively broad even without super thermals [4]. The broadening of emission regime for a particular frequency are expected to be higher for super thermals as the energy of the super thermals are much higher than that of the background electron. In other words even if a particular radial point have unique magnetic field it is capable of emitting a broad range of frequencies. Further, the the frequency intensity spectrum will also be affected by the relativistic frequency down shifting as $\gamma$ is greater than unity. Even if the frequency down shifting can be understood analytically the broadening of the emission drives the problem away from a simple analytical understanding. The problem will be further complicated by resonance, cutoff properties of thermal plasma, which it self can limit the range of frequencies reaching the detector. Thus it is very difficult to reach a logical conclusion on the exact influence of these parameters in producing the deviation without the numerical calculations. Figure 6 and figure 7 show the emission spectrum of super thermals obtained using the methodology explained above for the case with locations of super thermal at $q=1, q=1.5$ and $q=2$. The figures 6,7 shows that emission from the super thermal exist for almost all of the first harmonics frequencies. But the the intensity is more around the location of the super thermal and on the higher frequency side. It should be noted that no deviation is observed from the location of super thermals towards the detector side. This is because the corresponding frequencies are absorbed in between the location of emission and detector.

From the figures it can be noted that around two radial locations the deviation is relatively more. Firstly in the radial location where the super thermals are present. This is due to broaden first harmonics emission around the characteristic frequency which is downshifted. As stated above in the side towards the detector the error is shielded by the abortion in the region between the emission location and detector. Second peak exist in the high field side due to the downshifted second harmonic emission, again characterized by the relativistic broadening.

These results are explained in details in the subsequent subsections . In each subsections the effect of each parameters are analyzed and individually.

\subsection{Fraction of super thermals}

The fraction of the super thermals are defined as the ratio between the density of the super thermal electrons and background electron density. In this article the fraction is always expressed in percentage. The calculations are performed for six values of fractions such as $0.01 \%, 0.05 \%$, $0.1 \%, 0.2 \%, 0.4 \%$ and $0.5 \%$ for the case of super thermal location at $\mathrm{q}=1$. For the cases with super thermals at $\mathrm{q}=1.5$ and $\mathrm{q}=2$ the calculations are limited to three $0.1 \%$, 0.2 and 0.4. The Figure 6 shows deviation as radial function for different values of the fractions for the case with 

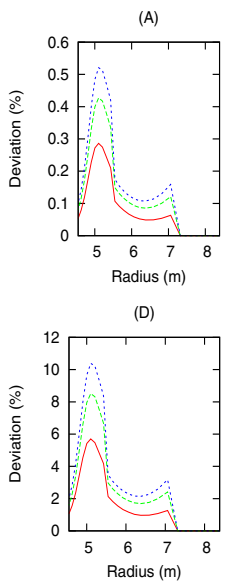

(B)
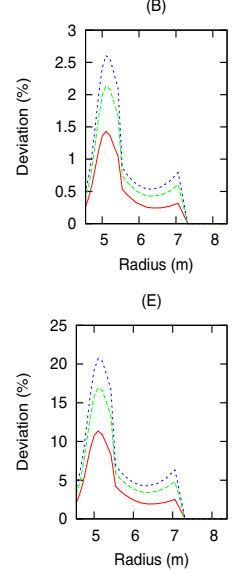
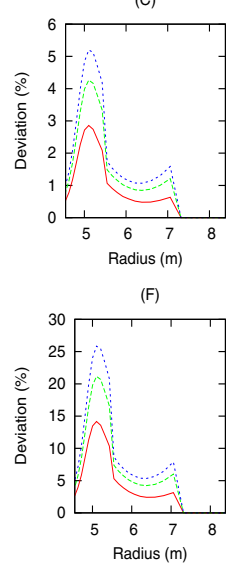

Figure 6. Deviation of temperature as a function of radius with super thermals for various fractions and energies.The location of the super thermal population is at $\mathrm{Q}=1$. In each plot three curves from bottom to top corresponds to $100 \mathrm{KeV}, 150 \mathrm{KeV}$ and $200 \mathrm{KeV}$ respectively. Different curves A,B,C,D,E and F corresponds to fraction of super thermals $0.01,0.05,0.1,0.2,0.4$ and 0.5 respectively.

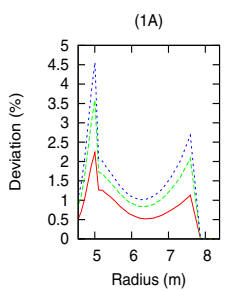

(2A)

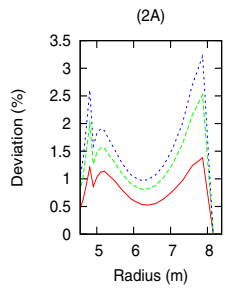

(1B)

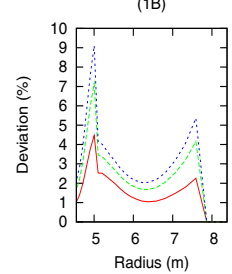

(2B)

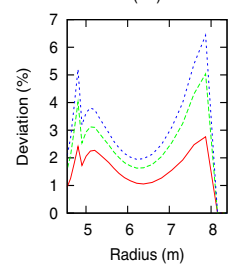

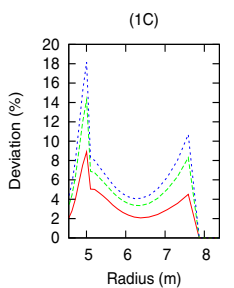

(2C)

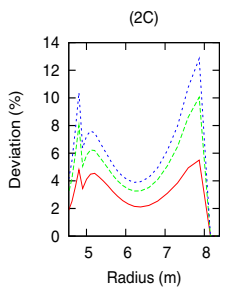

Figure 7. Deviation of temperature as a function of radius with super thermals for various fractions and energies. In each plot three curves from bottom to top corresponds to $100 \mathrm{KeV}, 150$ $\mathrm{KeV}$ and $200 \mathrm{KeV} .1 \mathrm{~A}: \mathrm{Q}=1.5$, Fraction $=0.1,1 \mathrm{~B}: \mathrm{Q}=1.5$, Fraction $=0.2,1 \mathrm{C}: \mathrm{Q}=1.5$, Fraction $=0.4,2 \mathrm{~A}: \mathrm{Q}=2$, Fraction $=0.1$, $2 \mathrm{~B}: \mathrm{Q}=2$, Fraction $=0.2,2 \mathrm{C}: \mathrm{Q}=2$, Fraction $=0.4$.

location of super thermal at $\mathrm{q}=1$. In each graph three different curves for different values of the energies are plotted together. Similar plots for the cases with location of the super thermals at $\mathrm{q}=1.5$ and $\mathrm{q}=2$ are presented in Figure 7. From these figures it can be noted that having more super thermals translates to more deviation, which is easy to understand as more super thermals results more emission.

Another interesting point can be noted from the figures as for same value of the energy and location of super thermals the deviation is scaling linearly with the fraction of the super thermals. This point is further examined in the

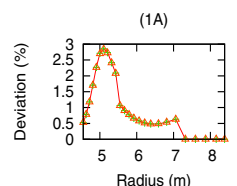

(2A)

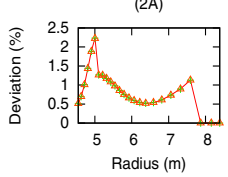

(3A)

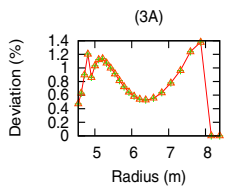

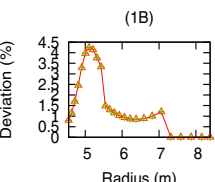

(2C)

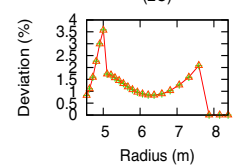

(3B)

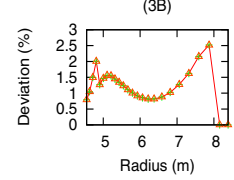

(1C)

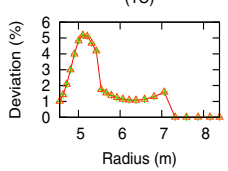

$(2 \mathrm{C})$

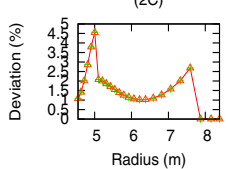

$(3 \mathrm{C})$

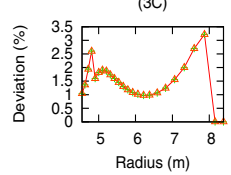

Figure 8. Deviation of radial temperature as a function of radius for various parameters. Curves $1 \mathrm{~A}, 1 \mathrm{~B}$ and $1 \mathrm{C}$ is for case $\mathrm{q}=1$ and energy $=100,150$ and 200 respectively. The three curves, red line is for frac $=0.1$, green dot is for Deviation divided by 2 frac $=0.2$ and triangle is for deviation divided by 4 for frac $=0.4$. Others curves $2 \mathrm{~A}, 2 \mathrm{~B}$, and $2 \mathrm{C}$ are for $\mathrm{q}=1.5$ while $3 \mathrm{~A}, 3 \mathrm{~B}$ and $3 \mathrm{C}$ corresponds to $\mathrm{q}=2$.

figure 8 . In figure 8 , for one value of energy and location of super thermals deviation are plotted as a function of the radial locations for three values of fractions $0.1 \%, 0.2 \%$ and $0.4 \%$. The deviations are divided by two and four for the cases with fractions equal to $0.2 \%$ and $0.4 \%$ respectively. This is to understand how the deviations are related with fractions of the super thermals. Nine such plots are shown for 3 X 3 values of energy and locations.

\subsection{Energy of super thermals}

Now we examine the effect of energy of the super thermals on the radial temperature measurement using ECE diagnostics. From the figures 6 and figure 7 it can be noted that increasing energy of super thermal increase the deviation in temperature. But the rate in increase decrease if we move towards higher energy values. In order to obtain a possible scaling law for energy of super thermals we can consider figure 9. In the figure 9 the ratio between deviations for different energies are plotted. We have considered ratio of deviation for three cases ie for $D e v_{100 \mathrm{KeV}} / \mathrm{Dev}_{150 \mathrm{KeV}}, \mathrm{Dev}_{150 \mathrm{KeV}} / \mathrm{Dev}_{200 \mathrm{KeV}}$ and $D e v_{100 \mathrm{KeV}} / \mathrm{Dev}_{200 \mathrm{KeV}}$. This is repeated for different values of fractions of the super thermals and location of the super thermals. In figure 9 , the plots differentiated by A,B and $\mathrm{C}$ corresponds to three different ratios and different curves (line,point and triangle) in each plot corresponds to different fractions. The graphs 2 and 3 are for radial locations $\mathrm{q}=1.5$ and $\mathrm{q}=2$ respectively. From the figure it should be noted that the ratio of deviation in temperature for two energies remain constant irrespective of fraction throughout the radial locations. This is true for all different values of fractions and radial locations scanned in this study. 


\subsection{Location of super thermals}

The simulations are repeated for 3 locations with $\mathrm{q}=1, \mathrm{q}=1.5$ and $\mathrm{q}=2$. Moving from $\mathrm{q}=1$ to $\mathrm{q}=2$ we move more towards detector which is placed near the wall at low field side. The deviation in temperature for corresponding cases are shown in Figure 6 and Figure 7. Following points can be noted from the figures.

- From all the figures the deviation is minimum near the center. This is have a physical significance as the super thermal are least interfering the central temperature measurement at least for the kind of super thermals studied here

- For the same values of all other parameters, for the cases with higher 'q' values deviation around the location of super thermals are increasing. In other words if the super thermals are present in the locations towards the wall at the low field side more deviation in the measured temperature around that region is noted. A possible explanation is given below. As we move away from the detector towards the high field side there is more chance that the radiation corresponding to the lower frequencies are getting absorbed between location of emission and detector.

- The peak near high field side almost remain same for the cases of $q=1$ and $q=1.5$ for same value of all other parameters. But the peak deviation in the high field side is considerably low for the case with location $q=2$.

No possible scaling law is obtained for this case.

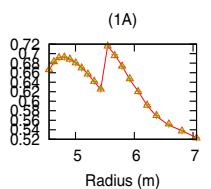

(2A)

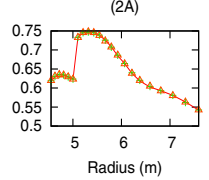

(3A)

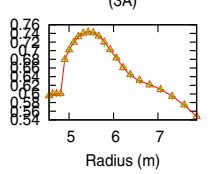

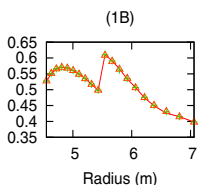

(2B)

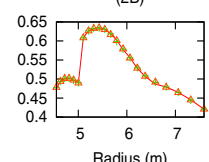

(3B)

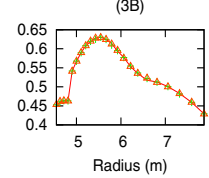

(1C)

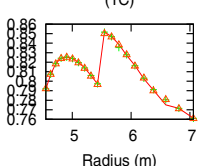

(2C)

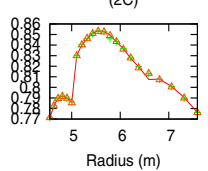

(3C)

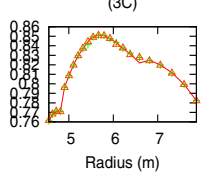

Figure 9. Deviation of radial temperature as a function of radial distance for various parameters. The curve $1 \mathrm{~A}$ shows ratio of deviation for energy $100 \mathrm{KeV}$ and $150 \mathrm{KeV}$ for all fractions ( line for frac $=0.1$ points for frac $=0.2$ and triangles for frac $=0.4$ ) for $\mathrm{Q}=1$. $1 \mathrm{~B}$ and $1 \mathrm{C}$ are for ratio of deviations for energies $150 \mathrm{KeV}$ and $200 \mathrm{KeV}$ and ratio between $100 \mathrm{KeV}$ and $200 \mathrm{KeV}$. Similar curves are shown in $2 \mathrm{~A}, 2 \mathrm{~B}$ and $2 \mathrm{C}$ for $\mathrm{Q}=1.5$ and in $3 \mathrm{~A}, 3 \mathrm{~B}$ and $3 \mathrm{C}$ for $\mathrm{q}=2$.

\section{References}

[1] M. Bornatici, R. Cano, O.D. Barbieri, F. Engelmann, Nucl. Fusion 23, 1153 (1983)

[2] E. de la Luna, J.E. Contributors, AIP Conference Proceedings 988, 63 (2008)

[3] P.V. Subhash, Y. Ghai, S.K. Amit, A.M. Begum, Comunnicated to Physics of Plasmas

[4] D. Bartlett, Physics issues of ECE and ECA for ITER, in Diagnostics for Experimental Thermonuclear Fusion Reactors, edited by P.E. Stott, G. Gorini, E. Sindoni (Plenum, New York and London, 1996), p. 183

[5] G.T. et al, Electron cyclotron emission measurements on high beta TFTR plasmas, in Proc. 9th Joint Workshop on ECE and ECRH, edited by J.Lohr (World Scientific, Singapore, 1995, 1995), p. 485

[6] E. de la Luna, V. Krivenski, G. Giruzzi, C. Gowers, R. Prentice, J.M. Travere, M. Zerbini, Review of Scientific Instruments 74, 1414 (2003)

[7] K.V. Beausang, S.L. Prunty, R. Scannell, M.N. Beurskens, M.J. Walsh, E. de La Luna, J.E. Contributors, Review of Scientific Instruments 82, 033514 ( 8) (2011)

[8] A. White, A. Hubbard, J. Hughes, P. Bonoli, M. Austin, A. Bader, R. Harvey, Y. Lin, Y. Ma, M. Reinke et al., Nuclear Fusion 52, 063021 (2012)

[9] R.M.J. Sillen, M. A.F.Allaart, W.J. Goedheer, A. Kattenberg (Rijnhuizen report, 1987)

[10] H.V.D. Brand, M.D. Baar, N.L. Cardozo, E.Westerhof, Nuclear Fusion 53, 013005 (2013)

[11] T. Casper, Y. Gribov, A. Kavin, V. Lukash, R. Khayrutdinov, H. Fujieda, C. Kessel, I. Organization, I.D. Agencies, Nuclear Fusion 54, 013005 (2014)

[12] A.R. Polevoi, S.Y. Medvedev, V.S. Mukhovatov, A.S. Kukushkin, Y. Murakami, M. Shimada, A.A. Ivanov, Journal of Plasma and Fusion Research SERIES 5, 82 (2002)

[13] I.P.B. Editors, I.P.E.G. Chairs, Co-Chairs, I.J.C. Team, P.I. Unit, Nuclear Fusion 39, 2137 (1999)

[14] S. Danani, H.K.B. Pandya, P. Vasu, M.E. Austin, Fusion Science and Technology 59, 651 (2011)

[15] M. Brusati, D. Bartlett, A. Ekedahl, P. Froissard, A. Airoldi, G. Ramponi, R.D. Silva, Y. Peysson, Nucl. Fusion 34, 23 (1994) 